atmosphere to force the gas through the boiling liquid, and at atmospheric pressure, one can employ a gas from a gasometer, gas cylinder or generator, according to the nature of the gas to be used.

\title{
A DEMONSTRATION OF THE WEIGHT OF A LITER OF CARBON DIOXIDE.
}

\section{By C. E. Linebarger.}

There are comparatively few quantitative experiments which are adapted for performance on the lecture table, as they require more care and attention to insure good results than can usually be given during the lecture. And yet the desirability of giving some quantitative work in the lecture room can hardly be questioned. A fundamental demonstration is the determination of the weight of a liter of a gas, and in schools where the laboratory work is purely qualitative, such a demonstration becomes almost peremptory. Carbon dioxide is a gas well suited for'such purposes. The methods which have been in vogue are, however, rather unsuitable for demonstration, although they may be made to yield satisfactory results in the laboratory as an individual experiment. The method described in this paper permits of the weighing of a liter of carbon dioxide with a satisfactory degree of accuracy, and in a reasonably short time, and further does not demand any protracted or delicate manipulation. If the class is familiar with the reduction of gas volumes to standard conditions, the demonstration from start to finish ought not to take more than half an hour, and the actual manipulation of the apparatus, apart from the weighings and measurement of the volume of the gas, does not require five minutes.

Recently there have been placed upon the market steel capsules* filled under considerable pressure with carbon dioxide.

* Their trade name is "Sparklets." 
They are manufactured for the purpose of making sparkling or soda water in small quantities, a bottle of special design being provided with a simple device to open the capsule and discharge the carbonic acid gas into the water with which the bottle is filled. The "pint size" of these capsules contains about two grams of carbon dioxide, and the capsules weigh about six grams each. With these capsules, the exact weight of about a liter of carbon dioxide may be found; the capsule is weighed, then opened so that the compressed gas may escape, and the capsule, now filled with air, reweighed.

The apparatus employed is shown in Fig. I. With the excep-

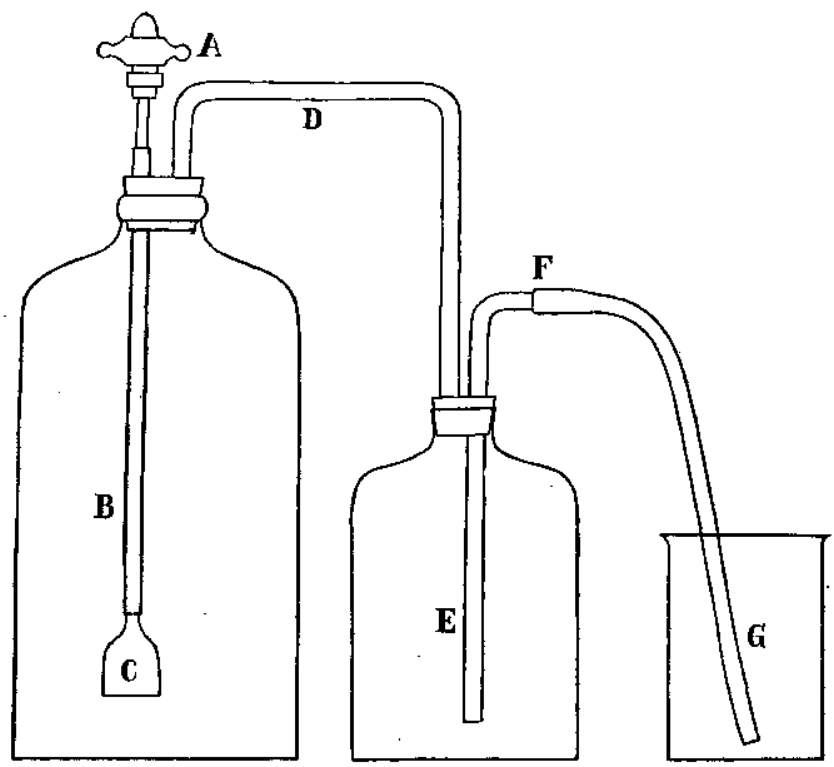

Fig. 1

tion of the "capsule opener," the parts of the apparatus are to be found in every laboratory.

B is a large bottle, the larger the better. An "acid bottle" will do, if the vertical branches of the tube $\mathrm{D}$ are as long as possible so as to make the space through which the carbon 
dioxide has to diffuse before reaching the water quite considerable. This bottle is fitted with a two-hole stopper, best of rtibber; through one hole passes the tube of the "capsule opener" A, and through the other a glass tube of wide bore, bent at right angle. A piece of rubber tubing is slipped over the lower end of the tube of the "capsule opener," to which is joined a little funnel $C$, made by drawing out a test tube. The object of the funnel is to allow the carbon dioxide to spread out a little on entering the bottle, and thus to decrease its speed somewhat. A smaller bottle, $\mathrm{E}$ (a two-liter size is good), is fitted with a two-hole stopper through which passes a wide tube making connections with $\mathrm{B}$, and an L-tube over which is slipped a piece of rubber tubing about $30 \mathrm{cms}$. long. $G$ is a beaker or bottle of about two inters capacity.

Before the demonstration is shown to the class, the apparatus should be set up all ready on the lecture table, $E$ filled with water, which is drawn over through $F$, so as to fill it also with water, and the end of $F$ kept under water in $G$, which is raised ap and supported by blocks so that the water will nearly fill $E$, and yet the levels in $G$ and $E$ will be the same. Especial care zust be exercised in pushing in the stoppers tightly and having all connections tight, as when the gas escapes from the capsule, it does so with a rush, and the pressure suddenly developed may force out the stoppers.

A capsule is weighed (to a centigram is sufficient), placed in the "opener," and the cap screwed down almost hard enough to pierce the capsule. The tip of the rubber tube in $G$ is then pinched together with the forefinger and thumb, and lifted up so that $G$ may be emptied and drained for a few seconds. The rubber tube is replaced in $\mathrm{G}$, care being taken to keep its tip always below the surface of the water. Without delay, the cap is screwed down (the "opener" being held with the little wrench) so that the pin punctures the capsule. As the gas escapes, it displaces the air in $\mathrm{B}$, which in turn displaces the water in $\mathrm{E}$. In this way the carbon dioxide does not come in contact with the water at all, or, at least, not for some time. As soon as no more water appears to flow into $G$, which is the case in a few 
seconds, it is lifted up so that the level of the water in it and in $\mathrm{E}$ are the same, and the apparatus is allowed to stand for a minute or so in order to bring the pressure in the apparatus to that of the atmosphere. In the meantime the thermometer (which had better be kept inside the large bottle all during the experiment) and barometer are read. No correction for aqueous tension has to be made, as the carbon dioxide is dry. The tip of the tube in $G$ is again closed with the thumb and the finger, and lifted out. The volume of the water in $G$ is found by pouring it out into a graduated cylinder or flask. All that remains to do now is to find the weight of the capsule emptied of carbon dioxide.

It is my practice to have the class work out the calculations for the reduction to standard conditions, which keeps them occupied, while I weigh the capsule. We then together calculate the weight of a liter of carbon dioxide from the data obtained. The values we have thus far found are 1.96, r.98, r.99, r.96, and I.98, which agree well with the accepted value, I.97.

Care must, of course, be taken to remove all the carbon dioxide from the large bottle, before making a second determination. This is readily accomplished by pouring the gas out, as if it were water.

Although this experiment has not been put into the hands of students to perform individually, there do not seem to be any good grounds for believing that it would not prove to be a good students' experiment. The apparatus does, indeed, take up considerable room, but several of them set up on a side table would suffice for a large class. One thing that certainly recommends the experiment for students' use is the great certainty that good results can be obtained even with comparatively careless handling. 